\title{
Solvability of boundary value problems of nonlinear fractional differential equations
}

Weiqi Chen ${ }^{1,2}$ and Yige Zhao ${ }^{3 *}$

${ }^{\text {*Correspondence: }}$

zhaoeager@126.com

${ }^{3}$ School of Control Science and Engineering, Shandong University, Jinan, Shandong 250061, P.R. China Full list of author information is available at the end of the article

\begin{abstract}
In this paper, we study the existence of multiple positive solutions for the nonlinear fractional differential equation boundary value problem $D_{0^{+}}^{\alpha} u(t)+f(t, u(t))=0$, $0<t<1, u(0)=u(1)=u^{\prime}(0)=0$, where $2<\alpha \leq 3$ is a real number, $D_{0^{+}}^{\alpha}$ is the Riemann-Liouville fractional derivative. By the properties of the Green's function, the lower and upper solution method and the Leggett-Williams fixed point theorem, some new existence criteria are established. As applications, examples are presented to illustrate the main results.

MSC: 34A08; 34B18

Keywords: fractional differential equation; boundary value problem; positive solution; fractional Green's function; fixed point theorem; lower and upper solution method
\end{abstract}

\section{Introduction}

Fractional differential equations have been of great interest. It is caused both by the intensive development of the theory of fractional calculus itself and by the applications. Apart from diverse areas of mathematics, fractional differential equations arise in rheology, dynamical processes in self-similar and porous structures, fluid flows, electrical networks, viscoelasticity, chemical physics, and many other branches of science; see [15]. Recently, there have appeared some papers dealing with the existence of solutions of fractional differential equations by the use of techniques of nonlinear analysis (fixed point theorems, Leray-Schauder theory, Adomian decomposition method, etc.); see [6-9]. Especially, boundary value problems for fractional differential equations have attracted considerable attention; see [10-31]. As is well known, the aim of finding solutions to boundary value problems is of main importance in various fields of applied mathematics. Recently, there seems to be a new interest in the study of the boundary value problems for fractional differential equations.

Bai and Lü [28] studied the following two-point boundary value problem of fractional differential equations:

$$
\begin{aligned}
& D_{0^{+}}^{\alpha} u(t)+f(t, u(t))=0, \quad 0<t<1, \\
& u(0)=u(1)=0,
\end{aligned}
$$

where $1<\alpha \leq 2$ is a real number and $D_{0^{+}}^{\alpha}$ is the standard Riemann-Liouville fractional derivative. They obtained the existence of positive solutions by means of the Krasnosel'skii fixed point theorem and the Leggett-Williams fixed point theorem.

\section{空 Springer}

(c) 2015 Chen and Zhao; licensee Springer. This is an Open Access article distributed under the terms of the Creative Commons Attribution License (http://creativecommons.org/licenses/by/4.0), which permits unrestricted use, distribution, and reproduction in any medium, provided the original work is properly credited. 
Zhang [29] considered the existence and multiplicity of positive solutions for the nonlinear fractional boundary value problem

$$
\begin{aligned}
& { }^{\mathrm{C}} D_{0^{+}}^{\alpha} u(t)=f(t, u(t)), \quad 0<t<1, \\
& u(0)+u^{\prime}(0)=0, u(1)+u^{\prime}(1)=0,
\end{aligned}
$$

where $1<\alpha \leq 2$ is a real number, $f:[0,1] \times[0,+\infty) \rightarrow[0,+\infty)$ is continuous and ${ }^{\mathrm{C}} D_{0^{+}}^{\alpha}$ is the standard Caputo fractional derivative. The author obtained the existence and multiplicity results of positive solutions by means of the Krasnosel'skii fixed point theorem and the Leggett-Williams fixed point theorem.

Liang and Zhang [30] investigated the following nonlinear fractional boundary value problem:

$$
\begin{aligned}
& D_{0^{+}}^{\alpha} u(t)+f(t, u(t))=0, \quad 0<t<1, \\
& u(0)=u^{\prime}(0)=u^{\prime \prime}(0)=u^{\prime \prime}(1)=0,
\end{aligned}
$$

where $3<\alpha \leq 4$ is a real number, $f \in C([0,1] \times[0,+\infty),(0,+\infty))$ and $D_{0^{+}}^{\alpha}$ is the standard Riemann-Liouville fractional derivative. By means of the lower and upper solution method and fixed point theorems, some results on the existence of positive solutions are obtained for the above fractional boundary value problems.

$\mathrm{Yu}$ and Jiang [31] discussed the following two-point boundary value problem of fractional differential equations:

$$
\begin{aligned}
& D_{0^{+}}^{\alpha} u(t)+f(t, u(t))=0, \quad 0<t<1, \\
& u(0)=u(1)=u^{\prime}(0)=0,
\end{aligned}
$$

where $2<\alpha \leq 3$ is a real number and $D_{0^{+}}^{\alpha}$ is the standard Riemann-Liouville fractional derivative. By the properties of the Green's function, they gave some results of multiple positive solutions for singular and nonsingular boundary value problems by means of the Leray-Schauder nonlinear alternative, a fixed point theorem on cones, and a mixed monotone method.

From the above works, we can see that, although the fractional boundary value problems have been investigated by some authors, the lower and upper solution method and the fixed point theorem due to Leggett-Williams are seldom considered. In addition, in the latter work the multiplicity of the solutions was not employed. Furthermore, the solution technique of upper and lower solutions was not studied, and it was also assumed that $2<\alpha \leq 3$. This paper will fill up the gap.

Motivated by all the works above, in this paper we discuss the boundary value problem

$$
\begin{aligned}
& D_{0^{+}}^{\alpha} u(t)+f(t, u(t))=0, \quad 0<t<1, \\
& u(0)=u(1)=u^{\prime}(0)=0,
\end{aligned}
$$

where $2<\alpha \leq 3$ is a real number and $D_{0^{+}}^{\alpha}$ is the Riemann-Liouville fractional differentiation. Using the lower and upper solution method and the Leggett-Williams fixed point 
theorem, we give some new existence criteria for the boundary value problem (1.1) and (1.2). Finally, we present an example to demonstrate our results.

The plan of the paper is as follows. In Section 2, we shall give some definitions and lemmas to prove our main results. In Section 3, we establish the existence of a single positive solution for the boundary value problem (1.1) and (1.2) by the lower and upper solution method. In Section 4, we establish the existence of multiple positive solutions for the boundary value problem (1.1) and (1.2) by the Leggett-Williams fixed point theorem. Examples are presented to illustrate the main results in Section 3 and Section 4, respectively. In Section 5, we give the conclusion of the paper.

\section{Preliminaries}

For the convenience of the reader, we give some background materials from fractional calculus theory to facilitate the analysis of problem (1.1) and (1.2). These materials can be found in the recent literature; see [31-33].

Definition 2.1 ([32]) The Riemann-Liouville fractional derivative of order $\alpha>0$ of a continuous function $f:(0,+\infty) \rightarrow \mathbb{R}$ is given by

$$
D_{0^{+}}^{\alpha} f(t)=\frac{1}{\Gamma(n-\alpha)}\left(\frac{d}{d t}\right)^{(n)} \int_{0}^{t} \frac{f(s)}{(t-s)^{\alpha-n+1}} d s
$$

where $n=[\alpha]+1,[\alpha]$ denotes the integer part of number $\alpha$, provided that the right side is pointwise defined on $(0,+\infty)$.

Definition 2.2 ([32]) The Riemann-Liouville fractional integral of order $\alpha>0$ of a func$\operatorname{tion} f:(0,+\infty) \rightarrow \mathbb{R}$ is given by

$$
I_{0^{+}}^{\alpha} f(t)=\frac{1}{\Gamma(\alpha)} \int_{0}^{t}(t-s)^{\alpha-1} f(s) d s
$$

provided that the right side is pointwise defined on $(0,+\infty)$.

From the definition of the Riemann-Liouville derivative, we can obtain the following statement.

Lemma 2.1 Let $\alpha>0$. If we assume $u \in C(0,1) \cap L^{1}(0,1)$, then the fractional differential equation

$$
D_{0^{+}}^{\alpha} u(t)=0
$$

has $u(t)=c_{1} t^{\alpha-1}+c_{2} t^{\alpha-2}+\cdots+c_{n} t^{\alpha-n}, c_{i} \in \mathbb{R}, i=1,2, \ldots, n$, as the unique solution, where $n$ is the smallest integer greater than or equal to $\alpha$.

Lemma 2.2 Assume that $u \in C(0,1) \cap L^{1}(0,1)$ with a fractional derivative of order $\alpha>0$ that belongs to $C(0,1) \cap L^{1}(0,1)$. Then

$$
I_{0^{+}}^{\alpha} D_{0^{+}}^{\alpha} u(t)=u(t)+c_{1} t^{\alpha-1}+c_{2} t^{\alpha-2}+\cdots+c_{n} t^{\alpha-n}, \quad \text { for some } c_{i} \in \mathbb{R}, i=1,2, \ldots, n,
$$

where $n$ is the smallest integer greater than or equal to $\alpha$. 
In the following, we present the Green's function of the fractional differential equation boundary value problem.

Lemma 2.3 ([31]) Let $h \in C[0,1]$ and $2<\alpha \leq 3$. The unique solution of problem

$$
\begin{aligned}
& D_{0+}^{\alpha} u(t)+h(t)=0, \quad 0<t<1, \\
& u(0)=u(1)=u^{\prime}(0)=0,
\end{aligned}
$$

is

$$
u(t)=\int_{0}^{1} G(t, s) h(s) d s
$$

where

$$
G(t, s)= \begin{cases}\frac{t^{\alpha-1}(1-s)^{\alpha-1}-(t-s)^{\alpha-1}}{\Gamma(\alpha)}, & 0 \leq s \leq t \leq 1, \\ \frac{t^{\alpha-1}(1-s)^{\alpha-1}}{\Gamma(\alpha)}, & 0 \leq t \leq s \leq 1 .\end{cases}
$$

Here $G$ is called the Green's function of the boundary value problem (2.1) and (2.2).

The following properties of the Green's function play important roles in this paper.

Lemma 2.4 ([31]) The function $G$ defined by (2.3) satisfies the following conditions:

(1) $G(t, s)=G(1-s, 1-t)$, for $t, s \in(0,1)$;

(2) $t^{\alpha-1}(1-t) s(1-s)^{\alpha-1} \leq \Gamma(\alpha) G(t, s) \leq(\alpha-1) s(1-s)^{\alpha-1}$, for $t, s \in(0,1)$;

(3) $G(t, s)>0$, for $t, s \in(0,1)$;

(4) $t^{\alpha-1}(1-t) s(1-s)^{\alpha-1} \leq \Gamma(\alpha) G(t, s) \leq(\alpha-1)(1-t) t^{\alpha-1}$, for $t, s \in(0,1)$.

Remark 2.1 Obviously, by Lemma 2.4, we have $u(t) \geq 0$ if $h(t) \geq 0$ on $t \in[0,1]$, where $u(t)$ and $h(t)$ are defined as (2.1).

Now we introduce the following two definitions concerned with the upper and lower solutions of the fractional boundary value problem (1.1) and (1.2).

Definition 2.3 A function $\beta$ is called a lower solution of the fractional boundary value problem (1.1) and (1.2), if $\beta \in C[0,1]$ and $\beta(t)$ satisfies

$$
\begin{aligned}
& -D_{0^{+}}^{\alpha} \beta(t) \leq f(t, \beta(t)), \quad 0<t<1,2<\alpha \leq 3, \\
& \beta(0) \leq 0, \quad \beta(1) \leq 0, \quad \beta^{\prime}(0) \leq 0,
\end{aligned}
$$

where $f \in C([0,1] \times[0,+\infty),(0,+\infty))$.

Definition 2.4 A function $\gamma$ is called a upper solution of the fractional boundary value problem (1.1) and (1.2), if $\gamma \in C[0,1]$ and $\gamma(t)$ satisfies

$$
\begin{aligned}
& -D_{0^{+}}^{\alpha} \gamma(t) \geq f(t, \gamma(t)), \quad 0<t<1,2<\alpha \leq 3, \\
& \gamma(0) \geq 0, \quad \gamma(1) \geq 0, \quad \gamma^{\prime}(0) \geq 0,
\end{aligned}
$$

where $f \in C([0,1] \times[0,+\infty),(0,+\infty))$. 
The following definition is about the nonnegative continuous concave functional.

Definition 2.5 The map $\theta$ is said to be a nonnegative continuous concave functional on a cone $P$ of a real Banach space $E$ provided that $\theta: P \rightarrow[0, \infty)$ is continuous and

$$
\theta(t x+(1-t) y) \geq t \theta(x)+(1-t) \theta(y)
$$

for all $x, y \in P$ and $0 \leq t \leq 1$.

The following lemma is fundamental in the proofs of our main results.

Lemma 2.5 ([33]) Let $P$ be a cone in a real Banach space $E, P_{c}=\{x \in P\|\| x \| \leq c\}, \theta$ a nonnegative continuous concave functional on $P$ such that $\theta(x) \leq\|x\|$, for all $x \in \bar{P}_{c}$, and $P(\theta, b, d)=\{x \in P \mid b \leq \theta(x),\|x\| \leq d\}$. Suppose $A: \bar{P}_{c} \rightarrow \bar{P}_{c}$ is completely continuous and there exist constants $0<a<b<d \leq c$ such that

(C1) $\{x \in P(\theta, b, d) \mid \theta(x)>b\} \neq \emptyset$ and $\theta(A x)>b$ for $x \in P(\theta, b, d)$;

(C2) $\|A x\|<a$ for $x \leq a$;

(C3) $\theta(A x)>b$ for $x \in P(\theta, b, c)$ with $\|A x\|>d$.

Then $A$ has at least three fixed points $x_{1}, x_{2}, x_{3}$ with

$$
\left\|x_{1}\right\|<a, \quad b<\theta\left(x_{2}\right), \quad a<\left\|x_{3}\right\| \quad \text { and } \quad \theta\left(x_{3}\right)<b .
$$

Remark 2.2 If we have $d=c$, then condition (C1) of Lemma 2.5 implies condition (C3) of Lemma 2.5 .

For convenience, we set $q(t)=t^{\alpha-1}(1-t), k(s)=s(1-s)^{\alpha-1}$, then

$$
q(t) k(s) \leq \Gamma(\alpha) G(t, s) \leq(\alpha-1) k(s) .
$$

\section{Single positive solution}

In this section, we establish the existence of single positive solution for the boundary value problem (1.1) and (1.2) by the lower and upper solution method. In this section, we set $f \in C([0,1] \times[0,+\infty),(0,+\infty))$. As an application, an example is given to illustrate the main results.

Lemma 3.1 If $u$ is a positive solution of (1.1) and (1.2), then there exist two constants $r$ and $R$ such that $r \rho(t) \leq u(t) \leq R \rho(t)$, where $\rho(t)=\int_{0}^{1} G(t, s) d s$.

Proof Since $u \in C[0,1]$, there exists $M^{\prime}>0$ so that $|u(t)| \leq M^{\prime}$ for $t \in[0,1]$. Taking

$$
r=\min _{(t, u) \in[0,1] \times\left[0, M^{\prime}\right]} f(t, u(t)), \quad R=\max _{(t, u) \in[0,1] \times\left[0, M^{\prime}\right]} f(t, u(t)) .
$$

In view of Lemma 2.3, we have

$$
r \int_{0}^{1} G(t, s) d s \leq u(t)=\int_{0}^{1} G(t, s) f(s, u(s)) d s \leq R \int_{0}^{1} G(t, s) d s
$$


By direct computation, we have

$$
\rho(t)=\int_{0}^{1} G(t, s) d s
$$

Thus we finish the proof of Lemma 3.1.

Theorem 3.1 The fractional boundary value problem (1.1) and (1.2) has a positive solution $u$ if the following conditions are satisfied:

$\left(\mathrm{H}_{f}\right) f(t, u) \in C\left([0,1] \times[0,+\infty), \mathbb{R}^{+}\right)$is nondecreasing relative to $u, f(t, \rho(t)) \not \equiv 0$ for $t \in$ $(0,1)$ and there exists a positive constant $\mu<1$ such that

$$
k^{\mu} f(t, u) \leq f(t, k u), \quad \forall 0 \leq k \leq 1
$$

Proof At first, we will prove that the functions $\beta(t)=k_{1} g(t), \gamma(t)=k_{2} g(t)$ are lower and upper solutions of (1.1) and (1.2), respectively, where $0<k_{1} \leq \min \left\{\frac{1}{a_{2}},\left(a_{1}\right)^{\frac{\mu}{1-\mu}}\right\}, k_{2} \geq$ $\max \left\{\frac{1}{a_{1}},\left(a_{2}\right)^{\frac{\mu}{1-\mu}}\right\}$,

$$
\begin{aligned}
& a_{1}=\min \left\{1, \inf _{t \in[0,1]} f(t, \rho(t))\right\}>0, \\
& a_{2}=\max \left\{1, \sup _{t \in[0,1]} f(t, \rho(t))\right\}
\end{aligned}
$$

and

$$
g(t)=\int_{0}^{1} G(t, s) f(s, \rho(s)) d s .
$$

In view of Lemma 2.3 and Remark 2.1, we know that $g(t)$ is a positive solution of the following problem:

$$
\begin{aligned}
& -D_{0^{+}}^{\alpha} g(t)=f(t, \rho(t)), \quad 0<t<1,2<\alpha \leq 3, \\
& g(0)=g(1)=g^{\prime}(0)=0 .
\end{aligned}
$$

From the conclusion of Lemma 3.1, we know that

$$
a_{1} \rho(t) \leq g(t) \leq a_{2} \rho(t), \quad t \in[0,1] .
$$

Thus, by virtue of the assumptions of Theorem 3.1, one shows that

$$
\begin{aligned}
& k_{1} a_{1} \leq \frac{\beta(t)}{\rho(t)} \leq k_{1} a_{2} \leq 1, \\
& \frac{1}{k_{2} a_{2}} \leq \frac{\rho(t)}{\gamma(t)} \leq \frac{1}{k_{2} a_{1}} \leq 1, \\
& \left(k_{1} a_{1}\right)^{\mu} \geq k_{1}, \quad\left(k_{2} a_{2}\right)^{\mu} \leq k_{2} .
\end{aligned}
$$


Therefore, we have

$$
\begin{aligned}
f(t, \beta(t)) & =f\left(t, \frac{\beta(t)}{\rho(t)} \rho(t)\right) \geq\left(\frac{\beta(t)}{\rho(t)}\right)^{\mu} f(t, \rho(t)) \\
& \geq\left(k_{1} a_{1}\right)^{\mu} f(t, \rho(t)) \geq k_{1} f(t, \rho(t)), \\
k_{2} f(t, \rho(t)) & =k_{2} f\left(t, \frac{\rho(t)}{\gamma(t)} \gamma(t)\right) \geq k_{2}\left(\frac{\rho(t)}{\gamma(t)}\right)^{\mu} f(t, \gamma(t)) \\
& \geq k_{2}\left(k_{2} a_{2}\right)^{-\mu} f(t, \gamma(t)) \geq f(t, \gamma(t)) .
\end{aligned}
$$

It implies that

$$
\begin{array}{ll}
-D_{0^{+}}^{\alpha} \beta(t)=k_{1} f(t, \rho(t)) \leq f(t, \beta(t)), & 0<t<1,2<\alpha \leq 3, \\
-D_{0^{+}}^{\alpha} \gamma(t)=k_{2} f(t, \rho(t)) \geq f(t, \gamma(t)), & 0<t<1,2<\alpha \leq 3 .
\end{array}
$$

Obviously, $\beta(t)=k_{1} g(t), \gamma(t)=k_{2} g(t)$ satisfy the boundary conditions (1.2). So, $\beta(t)=$ $k_{1} g(t), \gamma(t)=k_{2} g(t)$ are lower and upper solutions of (1.1) and (1.2), respectively.

Next, we will prove the fractional boundary value problem

$$
\begin{aligned}
& -D_{0^{+}}^{\alpha} u(t)=g(t, u(t)), \quad 0<t<1,2<\alpha \leq 3, \\
& u(0)=u(1)=u^{\prime}(0)=0
\end{aligned}
$$

has a solution, where

$$
g(t, u(t))= \begin{cases}f(t, \beta(t)), & \text { if } u(t) \leq \beta(t), \\ f(t, u(t)), & \text { if } \beta(t) \leq u(t) \leq \gamma(t), \\ f(t, \gamma(t)), & \text { if } \gamma(t) \leq u(t) .\end{cases}
$$

Thus, we consider that the operator $T: C[0,1] \rightarrow C[0,1]$ is defined as follows:

$$
T u(t)=\int_{0}^{1} G(t, s) g(s, u(s)) d s,
$$

where $G(t, s)$ is defined as (2.3). It is clear that $T$ is continuous in $C[0,1]$. Since the function $f(t, u)$ in nondecreasing in $u$, this shows that, for any $u \in C([0,1],[0,+\infty))$,

$$
f(t, \beta(t)) \leq g(t, u(t)) \leq f(t, \gamma(t)), \quad \text { for } t \in[0,1] .
$$

The operator $T: C[0,1] \rightarrow C[0,1]$ is continuous in view of continuity of $G(t, s)$ and $g(t, u(t))$. By means of the Arzela-Ascoli theorem, $T$ is a compact operator. Therefore, from the Leray-Schauder fixed point theorem, the operator $T$ has a fixed point, i.e., the fractional boundary value problem (3.1) has a solution.

Finally, we will prove that the fractional boundary value problem (1.1) and (1.2) has a positive solution.

Suppose that $u^{*}(t)$ is a solution of the fractional boundary value problem (3.1). Since the function $f(t, u)$ is nondecreasing in $u$, we know that

$$
f(t, \beta(t)) \leq g\left(t, u^{*}(t)\right) \leq f(t, \gamma(t)), \quad \text { for } t \in[0,1] .
$$


Thus,

$$
\begin{aligned}
& -D_{0^{+}}^{\alpha} z(t) \geq f(t, \gamma(t))-g\left(t, u^{*}(t)\right) \geq 0, \\
& z(0)=z(1)=z^{\prime}(0)=0,
\end{aligned}
$$

where $z(t)=\gamma(t)-u^{*}(t)$. By Remark 2.1, $z(t) \geq 0$, i.e., $u^{*}(t) \leq \gamma(t)$ for $t \in[0,1]$. Similarly, $\beta(t) \leq u^{*}(t)$ for $t \in[0,1]$. Therefore, $u^{*}(t)$ is a positive solution of the fractional boundary value problem (1.1) and (1.2). We have finished the proof of Theorem 3.1.

In the following, we present a simple example to explain our results.

Example 3.1 Consider the boundary value problem

$$
\begin{aligned}
& D_{0^{+}}^{\frac{5}{2}} u(t)+f(t, u)=0, \quad 0<t<1, \\
& u(0)=u(1)=u^{\prime}(0)=0
\end{aligned}
$$

and

$$
f(t, u)=t+u^{\mu}, \quad 0<\mu<1 .
$$

Proof Since $k^{\mu} \leq 1$ for $0<\mu<1$ and $0 \leq k \leq 1$. It is easy to verify that

$$
k^{\mu} f(t, u)=k^{\mu} t+k^{\mu} u^{\mu} \leq t+(k u)^{\mu}=f(t, k u) .
$$

Thus, by Theorem 3.1, we know that the boundary value problem (3.2) has a positive solution $u$.

\section{Multiple positive solutions}

In this section, we establish the existence of multiple positive solutions for the boundary value problem (1.1) and (1.2) by the Legget-Williams fixed point theorem. In this section, we set $f \in C([0,1] \times[0,+\infty),[0,+\infty))$. As an application, an example is given to illustrate the main results.

Let the Banach space $E=C[0,1]$ be endowed with the norm $\|u\|=\max _{0 \leq t \leq 1}|u(t)|$. Define the cone $P \subset E$ by

$$
P=\left\{u \in E: u(t) \geq \frac{q(t)}{\alpha-1}\|u\|, t \in[0,1]\right\} .
$$

Let the nonnegative continuous concave functional $\theta$ on the cone $P$ be defined by

$$
\theta(u)=\min _{\frac{1}{4} \leq t \leq \frac{3}{4}}|u(t)|
$$

Suppose that $u$ is a solution of the boundary value problem (1.1) and (1.2). Then

$$
u(t)=\int_{0}^{1} G(t, s) f(s, u(s)) d s, \quad t \in[0,1] .
$$


We define an operator $A: P \rightarrow E$ as follows:

$$
(A u)(t)=\int_{0}^{1} G(t, s) f(s, u(s)) d s, \quad t \in[0,1] .
$$

By Lemma 2.4, we have

$$
\begin{aligned}
& \|A u\| \leq \frac{1}{\Gamma(\alpha)} \int_{0}^{1}(\alpha-1) k(s) f(s, u(s)) d s \\
& (A u)(t) \geq \frac{1}{\Gamma(\alpha)} \int_{0}^{1} q(t) k(s) f(s, u(s)) d s \geq \frac{q(t)}{\alpha-1}\|A u\| .
\end{aligned}
$$

Thus, $A(P) \subset P$.

Then we have the following lemma.

Lemma 4.1 $A: P \rightarrow P$ is completely continuous.

Proof The operator $A: P \rightarrow P$ is continuous in view of the continuity of $G(t, s)$ and $f(t, u(t))$. By means of the Arzela-Ascoli theorem, $A: P \rightarrow P$ is completely continuous.

For convenience, we denote

$$
\begin{array}{ll}
M=\left(\frac{1}{\Gamma(\alpha)} \int_{0}^{1}(\alpha-1) k(s) d s\right)^{-1}, & N=\left(\frac{1}{\Gamma(\alpha)} \int_{\frac{1}{4}}^{\frac{3}{4}}(\alpha-1) \sigma k(s) d s\right)^{-1}, \\
\widetilde{N}=\left(\frac{1}{\Gamma(\alpha)} \int_{\frac{1}{4}}^{\frac{3}{4}}(\alpha-1) k(s) d s\right)^{-1}, & \sigma=\min _{\frac{1}{4} \leq t \leq \frac{3}{4}} \frac{q(t)}{\alpha-1} .
\end{array}
$$

Theorem 4.1 Suppose $f(t, u)$ is continuous on $[0,1] \times[0,+\infty)$ and there exist constants $0<a<b<c$ such that the following assumptions hold:

(B1) $f(t, u)<M a$, for $(t, u) \in[0,1] \times[0, a]$;

(B2) $f(t, u) \geq N b$, for $(t, u) \in[1 / 4,3 / 4] \times[b, c]$;

(B3) $f(t, u) \leq M c$, for $(t, u) \in[0,1] \times[0, c]$.

Then the boundary value problem (1.1) and (1.2) has at least three positive solutions $u_{1}, u_{2}$, $u_{3}$ with

$$
\begin{aligned}
& \max _{0 \leq t \leq 1}\left|u_{1}(t)\right|<a, \quad b<\min _{\frac{1}{4} \leq t \leq \frac{3}{4}}\left|u_{2}(t)\right|<\max _{0 \leq t \leq 1}\left|u_{2}(t)\right| \leq c, \\
& a<\max _{0 \leq t \leq 1}\left|u_{3}(t)\right| \leq c, \quad \min _{\frac{1}{4} \leq t \leq \frac{3}{4}}\left|u_{3}(t)\right|<b .
\end{aligned}
$$

Proof We show that all the conditions of Lemma 2.5 are satisfied.

If $u \in \bar{P}_{c}$, then $\|u\| \leq c$. Assumption (B3) implies $f(t, u(t)) \leq M c$ for $0 \leq t \leq 1$. Consequently,

$$
\begin{aligned}
\|A u\| & \leq \frac{1}{\Gamma(\alpha)} \int_{0}^{1}(\alpha-1) k(s) f(s, u(s)) d s \\
& <M c \frac{1}{\Gamma(\alpha)} \int_{0}^{1}(\alpha-1) k(s) d s=c=\|u\| .
\end{aligned}
$$


Hence, $A: \bar{P}_{c} \rightarrow \bar{P}_{c}$. In the same way, if $u \in \bar{P}_{a}$, then assumption (C2) of Lemma 2.5 is satisfied.

To check condition (C1) of Lemma 2.5, we choose $u(t)=(b+c) / 2,0 \leq t \leq 1$. It is easy to see that $u(t)=(b+c) / 2 \in P(\theta, b, c), \theta(u)=\theta((b+c) / 2)>b$, consequently, $\{u \in$ $P(\theta, b, c) \mid \theta(u)>b\} \neq \emptyset$. Hence, if $u \in P(\theta, b, c)$, then $b \leq u(t) \leq c$ for $1 / 4 \leq t \leq 3 / 4$. Thus,

$$
\begin{aligned}
\theta(A u) & =\min _{\frac{1}{4} \leq t \leq \frac{3}{4}}|(A u)(t)| \geq \frac{1}{\Gamma(\alpha)} \int_{\frac{1}{4}}^{\frac{3}{4}} q(t) k(s) f(s, u(s)) d s \\
& \geq \frac{1}{\Gamma(\alpha)} \int_{\frac{1}{4}}^{\frac{3}{4}}(\alpha-1) \sigma k(s) f(s, u(s)) d s \\
& \geq N b \frac{1}{\Gamma(\alpha)} \int_{\frac{1}{4}}^{\frac{3}{4}}(\alpha-1) \sigma k(s) d s=b,
\end{aligned}
$$

i.e., $\theta(A u)>b$ for all $u \in P(\theta, b, c)$. This shows that condition (C1) of Lemma 2.5 is satisfied.

By Lemma 2.5 and Remark 2.1, the boundary value problem (1.1) and (1.2) has at least three positive solutions $u_{1}, u_{2}$, and $u_{3}$, satisfying

$$
\begin{aligned}
& \max _{0 \leq t \leq 1}\left|u_{1}(t)\right|<a, \quad b<\min _{\frac{1}{4} \leq t \leq \frac{3}{4}}\left|u_{2}(t)\right|<\max _{0 \leq t \leq 1}\left|u_{2}(t)\right| \leq c, \\
& a<\max _{0 \leq t \leq 1}\left|u_{3}(t)\right| \leq c, \quad \min _{\frac{1}{4} \leq t \leq \frac{3}{4}}\left|u_{3}(t)\right|<b .
\end{aligned}
$$

The proof is complete.

Corollary 4.1 Suppose $f(t, u)$ is continuous on $[0,1] \times[0,+\infty)$ and there exist constants $0<a_{1} \leq \sigma b_{1}<(\alpha-1) \sigma b_{1}<b_{1}<c_{1}$ such that the following assumptions hold:

(B4) $f(t, u)<M a_{1}$, for $(t, u) \in[0,1] \times\left[0, a_{1}\right]$;

(B5) $f(t, u) \geq \tilde{N} b_{1}$, for $(t, u) \in[1 / 4,3 / 4] \times\left[\sigma b_{1}, c_{1}\right]$;

(B6) $f(t, u) \leq M c_{1}$, for $(t, u) \in[0,1] \times\left[0, c_{1}\right]$.

Then the boundary value problem (1.1) and (1.2) has at least three positive solutions $u_{1}, u_{2}$, $u_{3}$, satisfying

$$
\begin{aligned}
& \max _{0 \leq t \leq 1}\left|u_{1}(t)\right|<a_{1}, \quad \sigma b_{1}<\min _{\frac{1}{4} \leq t \leq \frac{3}{4}}\left|u_{2}(t)\right|<\max _{0 \leq t \leq 1}\left|u_{2}(t)\right| \leq c_{1}, \\
& a_{1}<\max _{0 \leq t \leq 1}\left|u_{3}(t)\right| \leq c_{1}, \quad \min _{\frac{1}{4} \leq t \leq \frac{3}{4}}\left|u_{3}(t)\right|<\sigma b_{1} .
\end{aligned}
$$

Proof If we choose $a=a_{1}, b=\sigma b_{1}$, and $c=c_{1}$, then from Theorem 4.1, the conclusion holds.

Theorem 4.2 If condition (B3) in Theorem 4.1 is replaced by

(B3') $\lim \sup _{u \rightarrow \infty} \frac{f(t, u)}{u}<M$.

Then the conclusion of Theorem 4.1 also holds.

Proof We only need to show there exists a number $c^{\prime}$ with $c^{\prime}>c$ and $A: \bar{P}_{c^{\prime}} \rightarrow \bar{P}_{c^{\prime}}$. 
From (B3'), we know there exist $R$ and $\varepsilon<M$, such that

$$
\frac{f(t, u)}{u} \leq \varepsilon, \quad u \geq R
$$

Let $L=\max _{u \in[0, R]} f(t, u), t \in[0,1]$.

In view of (4.1), it is easy to see that

$$
f(t, u) \leq L+\varepsilon u, \quad u \geq 0 .
$$

Now let $c^{\prime}$ be such that

$$
c^{\prime}>\max \left\{c, \frac{L}{M-\varepsilon}\right\} .
$$

Then for arbitrary $u \in \bar{P}_{c^{\prime}}, t \in[0,1]$, from (4.2) and (4.3), we obtain

$$
\begin{aligned}
\|A u\| & \leq \frac{1}{\Gamma(\alpha)} \int_{0}^{1}(\alpha-1) k(s) f(s, u(s)) d s \\
& \leq\left(L+\varepsilon c^{\prime}\right) \frac{1}{\Gamma(\alpha)} \int_{0}^{1}(\alpha-1) k(s) d s \leq c^{\prime} .
\end{aligned}
$$

Thus, $A: \bar{P}_{c^{\prime}} \rightarrow \bar{P}_{c^{\prime}}$.

Theorem 4.3 Suppose that there exist constants $0<a_{1}^{\prime}<\sigma b_{1}^{\prime}<(\alpha-1) \sigma b_{1}^{\prime}<b_{1}^{\prime}<c_{1}^{\prime}<a_{2}^{\prime}<$ $\sigma b_{2}^{\prime}<(\alpha-1) \sigma b_{2}^{\prime}<b_{2}^{\prime}<c_{2}^{\prime}<\cdots<a_{n}^{\prime}, n \in \mathbb{N}$, for $i=1,2, \ldots, n$, such that

(B7) $f(t, u)<M a_{i}^{\prime}$, for $(t, u) \in[0,1] \times\left[0, a_{i}^{\prime}\right]$;

(B8) $f(t, u) \geq \tilde{N} b_{i}^{\prime}$, for $(t, u) \in[1 / 4,3 / 4] \times\left[\sigma b_{i}^{\prime}, c_{i}^{\prime}\right]$.

Then the boundary value problem (1.1) and (1.2) has at least $2 n-1$ positive solutions.

Proof When $n=1$, it is immediate from condition (B7) that $A: \bar{P}_{a_{1}^{\prime}} \rightarrow \bar{P}_{a_{1}^{\prime}}$, which means that $A$ has at least one point $u_{1} \in \bar{P}_{a_{1}^{\prime}}$ by the Schauder fixed point theorem.

When $n=2$, it is clear that Corollary 4.1 holds (with $c^{\prime}=a_{2}^{\prime}$ ). Then we can obtain at least three positive solutions $u_{1}, u_{2}$, and $u_{3}$, satisfying

$$
\begin{aligned}
& \max _{0 \leq t \leq 1}\left|u_{1}(t)\right|<a_{1}^{\prime}, \quad \sigma b_{1}^{\prime}<\min _{\frac{1}{4} \leq t \leq \frac{3}{4}}\left|u_{2}(t)\right|<\max _{0 \leq t \leq 1}\left|u_{2}(t)\right| \leq c_{1}^{\prime}, \\
& a_{1}^{\prime}<\max _{0 \leq t \leq 1}\left|u_{3}(t)\right| \leq c_{1}^{\prime} \quad \text { and } \min _{\frac{1}{4} \leq t \leq \frac{3}{4}}\left|u_{3}(t)\right|<\sigma b_{1}^{\prime} .
\end{aligned}
$$

In this way, we finish the proof by induction. The proof is complete.

In the following, we present a simple example to illustrate our results.

Example 4.1 Consider the boundary value problem

$$
\begin{aligned}
& D_{0^{+}}^{\frac{5}{2}} u(t)+f(t, u)=0, \quad 0<t<1, \\
& u(0)=u(1)=u^{\prime}(0)=0,
\end{aligned}
$$


where

$$
f(t, u)= \begin{cases}\frac{t}{10}+177 u^{2}, & \text { for } u \leq 1 \\ 176+\frac{t}{10}+u, & \text { for } u>1\end{cases}
$$

We have

$$
\begin{aligned}
& M=\left(\frac{1}{\Gamma(\alpha)} \int_{0}^{1}(\alpha-1) k(s) d s\right)^{-1}=\frac{35 \sqrt{\pi}}{8} \approx 13.7375, \\
& N=\left(\frac{1}{\Gamma(\alpha)} \int_{\frac{1}{4}}^{\frac{3}{4}}(\alpha-1) \sigma k(s) d s\right)^{-1} \approx 176.801 .
\end{aligned}
$$

Choosing $a=\frac{1}{12}, b=1, c=14$, we have

$$
\begin{aligned}
& f(t, u)=\frac{t}{10}+177 u^{2} \leq 1.3292 \leq M a \approx 1.37375, \quad \text { for }(t, u) \in[0,1] \times\left[0, \frac{1}{12}\right], \\
& f(t, u)=176+\frac{t}{10}+u \geq 177.025 \geq N b \approx 176.801, \quad \text { for }(t, u) \in[1 / 4,3 / 4] \times[1,14], \\
& f(t, u)=176+\frac{t}{10}+u \leq 190.1 \leq M c \approx 192.325, \quad \text { for }(t, u) \in[0,1] \times[0,14] .
\end{aligned}
$$

From Theorem 4.1, the boundary value problem (4.4) and (4.5) has at least three positive solutions $u_{1}, u_{2}, u_{3}$ satisfying

$$
\begin{aligned}
& \max _{0 \leq t \leq 1}\left|u_{1}(t)\right|<\frac{1}{12}, \quad 1<\min _{\frac{1}{4} \leq t \leq \frac{3}{4}}\left|u_{2}(t)\right|<\max _{0 \leq t \leq 1}\left|u_{2}(t)\right| \leq 14, \\
& \frac{1}{12}<\max _{0 \leq t \leq 1}\left|u_{3}(t)\right| \leq 14, \quad \min _{\frac{1}{4} \leq t \leq \frac{3}{4}}\left|u_{3}(t)\right|<1 .
\end{aligned}
$$

\section{Conclusions}

In this paper, we have studied the existence of positive solutions for a boundary value problem of nonlinear fractional differential equations involving the Riemann-Liouville fractional derivative. The existence of a single positive solution for the given problem has been obtained by using the properties of the Green's function and the lower and upper solution method, while the existence of multiple positive solutions is based on the Leggett-Williams fixed point theorem. The main results are well illustrated with the help of examples. Our results improve the work presented in [31].

The authors declare that they have no competing interests.

\section{Authors' contributions}

The authors declare that the study was realized in collaboration with the same responsibility. All authors read and approved the final manuscript.

\section{Author details}

'School of Economics, Shandong University, Jinan, Shandong 250100, P.R. China. ${ }^{2}$ School of Physical Education, Shandong University, Jinan, Shandong 250061, P.R. China. ${ }^{3}$ School of Control Science and Engineering, Shandong University, Jinan, Shandong 250061, P.R. China. 


\section{Acknowledgements}

The authors sincerely thank the reviewers for their valuable suggestions and useful comments that have led to the present improved version of the original manuscript. This research is supported by the National Natural Science Foundation of China (G61374065, G61374002), and the Research Fund for the Taishan Scholar Project of Shandong Province of China.

\section{Received: 8 October 2014 Accepted: 13 January 2015 Published online: 31 January 2015}

\section{References}

1. Miller, KS, Ross, B: An Introduction to the Fractional Calculus and Fractional Differential Equation. Wiley, New York (1993)

2. Oldham, KB, Spanier, J: The Fractional Calculus. Academic Press, New York (1974)

3. Podlubny, I: Fractional Differential Equations. Academic Press, New York (1999)

4. Samko, SG, Kilbas, AA, Marichev, Ol: Fractional Integral and Derivative: Theory and Applications. Gordon \& Breach, Yverdon (1993)

5. Agarwal, RP: Formulation of Euler-Lagrange equations for fractional variational problems. J. Math. Anal. Appl. 272, 368-379 (2002)

6. Delbosco, D, Rodino, L: Existence and uniqueness for a nonlinear fractional differential equation. J. Math. Anal. Appl. 204, 609-625 (1996)

7. Zhang, S: The existence of a positive solution for nonlinear fractional differential equation. J. Math. Anal. Appl. 252, 804-812 (2000)

8. Zhang, S: Existence of positive solutions for some class of nonlinear fractional equation. J. Math. Anal. Appl. 278 136-148 (2003)

9. Jafari, H, Daftardar-Gejii, V: Positive solutions of nonlinear fractional boundary value problems using Adomian decomposition method. Appl. Math. Comput. 180, 700-706 (2006)

10. $\mathrm{Xu}, \mathrm{X}$, Jiang, $\mathrm{D}$, Yuan, $\mathrm{C}$ : Multiple positive solutions for the boundary value problem of a nonlinear fractional differential equation. Nonlinear Anal. 71, 4676-4688 (2009)

11. Feng, W, Sun, S, Han, Z, Zhao, Y: Existence of solutions for a singular system of nonlinear fractional differential equations. Comput. Math. Appl. 62, 1370-1378 (2011)

12. Sun, $S, Z$ hao, $Y$, Han, Z, Xu, M: Uniqueness of positive solutions for boundary value problems of singular fractional differential equations. Inverse Probl. Sci. Eng. 20(3), 299-309 (2012)

13. Zhao, Y, Sun, S, Han, Z, Feng, W: Positive solutions for a coupled system of nonlinear differential equations of mixed fractional orders. Adv. Differ. Equ. 2011, 10 (2011)

14. Zhao, Y, Sun, S, Han, Z, Li, Q: Theory of fractional hybrid differential equations. Comput. Math. Appl. 62, 1312-1324 (2011)

15. Qiu, T, Bai, Z: Existence of positive solutions for singular fractional equations. Electron. J. Differ. Equ. $2008,146(2008)$

16. Ahmad, B, Nieto, JJ: Boundary value problems for a class of sequential integrodifferential equations of fractional order. J. Funct. Spaces Appl. 2013, Article ID 149659 (2013)

17. Jiang, D, Yuan, C: The positive properties of the Green function for Dirichlet-type boundary value problems of nonlinear fractional differential equations and its application. Nonlinear Anal. TMA 72, 710-719 (2010)

18. Zhao, Y, Sun, S, Han, Z, Li, Q: Positive solutions to boundary value problems of nonlinear fractional differential equations. Abstr. Appl. Anal. 2011, Article ID 390543 (2011)

19. Zhao, Y, Sun, S, Han, Z, Li, Q: The existence of multiple positive solutions for boundary value problems of nonlinear fractional differential equations. Commun. Nonlinear Sci. Numer. Simul. 16, 2086-2097 (2011)

20. Zhao, Y, Sun, S, Han, Z, Zhang, M: Positive solutions for boundary value problems of nonlinear fractional differential equations. Appl. Math. Comput. 217, 6950-6958 (2011)

21. Ahmad, B, Ntouyas, SK: On higher-order sequential fractional differential inclusions with nonlocal three-point boundary conditions. Abstr. Appl. Anal. 2014, Article ID 659405 (2014)

22. Ahmad, B, Agarwal, RP: Some new versions of fractional boundary value problems with slit-strips conditions. Bound. Value Probl. 2014, 175 (2014)

23. Ahmad, B, Nieto, JJ: A class of differential equations of fractional order with multi-point boundary conditions. Georgian Math. J. 21, 243-248 (2014)

24. Zhang, X, Liu, L, Wu, Y, Lu, Y: The iterative solutions of nonlinear fractional differential equations. Appl. Math. Comput. 219, 4680-4691 (2013)

25. Zhang, $X, L i u, L, W u, Y$ : Existence results for multiple positive solutions of nonlinear higher order perturbed fractional differential equations with derivatives. Appl. Math. Comput. 219, 1420-1433 (2012)

26. Zhang, $X$, Liu, L, Wu, Y: Multiple positive solutions of a singular fractional differential equation with negatively perturbed term. Math. Comput. Model. 55, 1263-1274 (2012)

27. Wang, Y, Liu, L, Wu, Y: Positive solutions for a nonlocal fractional differential equation. Nonlinear Anal. 74, 3599-3605 (2011)

28. Bai, Z, Lü, H: Positive solutions for boundary value problem of nonlinear fractional differential equation. J. Math. Anal. Appl. 311, 495-505 (2005)

29. Zhang, S: Positive solutions for boundary-value problems of nonlinear fractional differential equations. Electron. J. Differ. Equ. 2006, 36 (2006)

30. Liang, S, Zhang, J: Positive solutions for boundary value problems of nonlinear fractional differential equation Nonlinear Anal. TMA 71, 5545-5550 (2009)

31. Yu, Y, Jiang, D: Multiple Positive Solutions for the Boundary Value Problem of A Nonlinear Fractional Differential Equation. Northeast Normal University (2009)

32. Kilbas, AA, Srivastava, HH, Trujillo, JJ: Theory and Applications of Fractional Differential Equations. Elsevier, Amsterdam (2006)

33. Leggett, RW, Williams, LR: Multiple positive fixed points of nonlinear operators on ordered Banach spaces. Indiana Univ. Math. J. 28, 673-688 (1979) 